\title{
ABELIAN VARIETIES OVER FUNCTION FIELDS
}

\author{
BY \\ WEI-LIANG CHOW
}

1. Introduction. Let $K$ be a field and $U$ be a variety defined over $K$; let $u$ be a generic point of $U$ over $K$, so that $K(u)$ is a regular extension of $K$. Let $W$ be another variety defined over $K$, and let $A_{u}$ be a simple subvariety in $W$ which is an Abelian variety defined over $K(u)$; then there exists (Weil [5, Chap. VII, Theorem 12 (iii)]) in the product variety $U \times W$ a subvariety $T$, defined over $K$, such that $T \cdot(u \times W)=u \times A_{u}$. As we shall see later $(\$ 3$, Lemma 2 and the last part of the proof of Theorem 3), there exists a bunch $B$. of subvarieties in $U$, normally algebraic over $K$, such that the intersection $T \cdot\left(u_{0} \times W\right)$ is defined for every point $u_{0}$ in $U-B$ and is an Abelian variety defined over $K\left(u_{0}\right)$. Thus the Abelian variety $A_{u}$ generates over the field $K$ an algebraic system $\left\{A_{u}\right\}$ of Abelian varieties in $W$, parametrized by the open variety $U-B$, and $A_{u}$ is a generic element over $K$ of this algebraic system. In this paper we shall deal with the problem of finding geometrical entities which are associated with an algebraic system of Abelian varieties in a birationally invariant way and hence can be regarded as birational invariants of such a system; more specifically, since we are interested mainly in Abelian varieties here, we shall investigate whether there are Abelian varieeties which are invariantly associated with such a system. It is clear that any birational invariant of the parameter variety $U$, or the graph $T$, or the carrier variety of the system (which is the projection of $T$ into $W$ ), is also a birational invariant of the algebraic system $\left\{A_{u}\right\}$; however, since these are invariants of algebraic systems in general, not specifically invariants of algebraic systems of Abelian varieties, we shall not consider them here, though there are some interesting relations between these invariants and those which will be considered. Instead, we shall consider here some invariants which arise specifically from the fact that we are dealing with a system of Abelian varieties. The main purpose of this paper is to show that, to every algebraic system of Abelian varieties, defined over a field $K$, it is possible to attach in a birationally invariant way two Abelian varieties, which are defined over $K$ and are isogeneous to each other over $K$. These two Abelian varieties will be called the $K$-image and the $K$-trace of the generic Abelian variety $A_{u}$ of the algebraic system; they will play an important role in our abstract theory of Picard varieties, which will be presented in a forthcoming paper.

We begin with a few remarks on terminology. We shall follow in general the terminology of Weil, as developed in his books [5] and [6], with the following modifications. First, we shall deal only with points and varieties in

Received by the editors October 22, 1953. 
projective spaces; thus, in particular, all Abelian varieties can be considered as embedded in some one projective space, so that, for example, we can without any loss of generality replace the variety $W$ in the preceding paragraph by a projective space. In fact, this restriction to varieties in a projective space is used in an essential way only in the proof of Theorem 3; moreover, in view of the fact (which will be proved in our forthcoming paper on Picard varieties) that any Abelian variety is regularly isomorphic to an Abelian variety in a projective space, defined over the same field as the original one, this restriction entails in reality no loss of generality for our results. Next, we shall use the expression "extension" of a field in its usual general sense, not necessarily meaning a finitely generated extension, as is the case with Weil; however, whenever we speak of an extension $K(u)$ or $K(v)$ of a field $K$, this is to indicate that $K(u)$ or $K(v)$ is the (finitely generated) extension obtained from $K$ by the adjunction of a point $u$ or $v$ in some projective space. We shall say that an extension $K^{*}$ of $K$ is separably generated if it preserves $p$-independence, $p$ being the characteristic of $K$ (MacLane $[4, \S 4]$ ); and we shall say that an extension $K^{*}$ of $K$ is primary, if $K^{*} \cap \bar{K}$ is a purely inseparable extension of $K$. An extension which is both separably generated and primary is called regular, and one sees readily that this definition agrees with that of Weil in case of a finitely generated extension. Another modification is that we shall use the expressions "homomorphism" and "isomorphism" in their strictly group theoretic sense. We shall say that a homomorphism $H$ of an Abelian variety $A$ into another Abelian variety $B$ is rational if $H$ is also a rational transformation of $A$ into $B$ (so that it is a homomorphism in the terminology of Weil), and we shall say that a rational homomorphism $H$ is regular if, $K$ being a field of definition for $A, B, H$, and $x$ being a generic point of $A$ over $K$, the field $K(x)$ is a regular extension of $K(H(x))$. It is clear that a rational isomorphism is regular if and only if it is birational. Finally, for the sake of convenience, we shall use sometimes the expression "almost every" in the following sense: Let $U$ be a variety defined over a field $K$, or more generally a prime rational cycle over $K$, and let $u$ be a generic point of $U$ over $K$; we shall say that a property holds for almost every specialization of $u$ over $K$ if there is a bunch $B$ of proper subvarieties in $U$ such that the property holds for every point in $U-B$.

We shall assume that the reader is familiar with the theory of associated forms of positive cycles in a projective space, as developed in Chow-van der Waerden [3], and we shall denote by $M\left(S_{n} ; r, d\right)$ the bunch of varieties in a projective space which consists of the associated points of all positive cycles of dimension $r$ and degree $d$ in the projective space $S_{n}$ of $n$ dimensions. We shall also use the terminology and results of our note [2], except that we shall use the expression "Abelian function field" instead of "Abelian field"; this note was originally a part of the present paper, but was published separately on account of its independent interest. 
In $\$ 2$, we shall prove the fact, well known in the classical case, that an algebraic system of Abelian varieties (of positive dimension) cannot exist in an Abelian variety; in $\$ 3$, we shall show that the generic element $A_{u}$ of an algebraic system of Abelian varieties, defined over a field $K$, is regularly isomorphic to an Abelian variety defined over $K$ if and only if every two generic elements in the algebraic system are regularly isomorphic to each other. On the basis of these results, or rather somewhat more general versions of these results proved in $\$ \S 2$ and 3 , we shall prove in the next two sections the existence of the $K$-image and the $K$-trace. In $\S 4$, we shall consider rational homomorphisms of the Abelian variety $A_{u}$ into Abelian varieties defined over $K$, and we shall show that among all such rational homomorphisms there exists one which has the "universal mapping" property in the sense that any rational homomorphism of $A_{u}$ into any Abelian variety defined over $K$ must "go through" this one. The image Abelian variety of $A_{u}$ under this rational homomorphism is then defined as the $K$-image of $A_{u}$ over $K(u)$; it is uniquely determined up to a regular isomorphism over $K$. $\ln \S 5$, we shall consider dually rational homomorphisms (or isomorphisms) of Abelian varieties defined over $K$ into the Abelian variety $A_{u}$, and we shall show that among all such rational homomorphisms there exists one (which is an isomorphism) which has the "universal mapping" property in the sense that any rational homomorphism of any Abelian variety defined over $K$ into $A_{u}$ must "go through" this one. The Abelian variety defined over $K$ which is mapped by this isomorphism into $A_{u}$ is called the $K$-trace of $A_{u}$ over $K(u)$; it is uniquely determined up to a regular isomorphism over $K$. The $K$-trace is independent of any extension of the ground field $K$, while the $K$-image remains unchanged up to an isomorphism under any such extension, though the isomorphism involved might not be regular (see however the remarks at the end of $\$ 5$ ). These two invariants of the algebraic system $\left\{A_{u}\right\}$, the $K$-image and the $K$-trace, are connected by a relation of isogeneity, which is derived from the application of the Poincaré Complete Reducibility Theorem to the Abelian variety $A_{u}$ and its $K$-image.

The concepts of the $K$-image and the $K$-trace arise in a natural way from the theory of Picard varieties. Let $V$ be a variety of dimension $r$ in a projective space $S_{n}$, defined over a field $K$, which has no singular subvarieties of dimension $r-1$, and consider the algebraic system of curves on $V$ generated over $K$ by the intersection curve $C_{u}$ of a generic linear subspace (over $K$ ) of dimension $n-r+1$ in $S_{n}$, where $K(u)$ is a purely transcendental extension of $K$. Since the curve $C_{u}$ is defined over $K(u)$, the Jacobian variety $J_{u}$ of $C_{u}$ is also defined over $K(u)$, according to a result of ours proved elsewhere. In our paper [1], we have shown that, in the classical case, the $K$-image of $J_{u}$ over $K(u)$ is the Albanese variety of $V$ and the $K$-trace of $J_{u}$ over $K(u)$ is the Picard variety of $V$; as we have mentioned there, an independent proof of the existence of the $K$-image and the $K$-trace will enable us to dispense with the 
classical construction of the Albanese and Picard varieties by means of the periods of Abelian integrals and thus serve as a starting point of a purely algebraic theory of the Picard varieties over an arbitrary ground field. This algebraic theory of Picard varieties will be presented in a forthcoming paper.

2. Algebraic subgroups in an Abelian variety. Let $V$ be a variety and $A$ be an Abelian variety, both defined over a field $K$, and let $F$ be a rational transformation of $V$ into $A$, defined over a purely transcendental extension $K(u)$ of $K$, where $u$ is a generic point over $K$ of a projective space $S$. If $x$ is a generic point of $V$ over $K(u)$, then the correspondence $u \rightarrow F(x)$ defines a rational transformation of $S$ into $A$, defined over $K(x)$; according to [6, Theorem 8, Corollary], the point $F(x)$ is independent of $u$ and hence is rational over $K(x)$. Since $K(x)$ is linearly disjoint with respect to $K(u)$, it follows that $K(F(x))$ is also linearly disjoint with respect to $K(u)$; this shows that the rational transformation $F$ is defined over $K$ and hence the subvariety $F(V)$ in $A$ is also defined over $K$.

If $K(u)$ is not a purely transcendental extension of $K$, then the above statement will not be true in general; however, we shall show that if $V$ is also an Abelian variety and if $F$ is a rational homomorphism of $V$ into $A$, then it is still true that $F$ is defined over $K$ and hence the image Abelian subvariety $F(V)$ in $A$ is also defined over $K$, provided that $K(u)$ is a primary extension of $K$. Furthermore, even if the Abelian variety $V$ is only defined over $K(u)$, it is still true that the Abelian subvariety $F(V)$ in $A$ is defined over $K$, though of course the homomorphism $F$ will be in general defined only over $K(u)$. Both these results are consequences of a general theorem which asserts that any Abelian subvariety in $A$ which is defined over $K(u)$ is also defined over $K$, a theorem which expresses essentially the fact that an Abelian variety cannot contain a continuous system of Abelian subvarieties or, more generally, algebraic subgroups. In case of complex ground field, this theorem is a simple consequence of the fact that an analytic subgroup of a complex torus is uniquely determined by a subgroup of its fundamental group, which is a discrete group. In case of an arbitrary ground field, this argument is of course not available; however, a substitute for this can be found in the fact that to each Abelian subvariety $B$ in an Abelian variety $A$ there exists an endomorphism of $A$ which maps $A$ onto $B$ (Weil [6, Proposition 25]), and the fact that the module of endomorphisms of $A$ is a finite module over the rational integers (Weil [6, Theorem 37]). We shall give here a simple direct proof of this theorem, which is entirely elementary in character.

Theorem 1. Let $A$ be an Abelian variety; let $K$ be a field of definition for $A$ and let $K^{*}$ be an extension of $K$. If an algebraic subgroup $X$ in $A$ is normally algebraic over $K^{*}$, then it is normally algebraic over $K^{*} \cap \bar{K}$.

Proof. Without any loss of generality, we can assume that $K^{*}=K(u)$ is a finitely generated extension, for otherwise we can replace $K^{*}$ by $K^{*} \cap K_{1}$, 
where $K_{1}$ is a finitely generated extension of $K$ over which $X$ is defined. It is sufficient to show that $X$ is defined over $\bar{K}$, for then $X$ must be normally algebraic over $K^{*} \cap \bar{K}$; we can therefore assume that $K$ is algebraically closed, so that $K(u)$ is a regular extension of $K$. Furthermore, we can also assume that $X$ is an Abelian variety, for an algebraic subgroup in $A$ is algebraic over $K$ if and only if the maximal Abelian variety contained in it is algebraic over $K$. Let $U$ be the variety which is the locus of $u$ over $K$, and let $x$ be a generic point of $X$ over $K(u)$; then the point $u \times x$ in $U \times A$ has as its locus over $K$ a subvariety $T$ in $U \times A$. If $u^{\prime} \times x^{\prime}$ is a generic point of $T$ over $K$, independent with respect to $u \times x$ over $K$, then the point $x^{\prime}$ has a locus $X^{\prime}$ over $K\left(u^{\prime}\right)$, which is an Abelian subvariety in $A$ and has the same dimension $d$ as that of $X$. Let $X^{\prime \prime}$ be the locus of the point $x+x^{\prime}$ over $K\left(u, u^{\prime}\right)$; it is clear that $X^{\prime \prime}$ is an Abelian subvariety in $A$ and is the subgroup in $A$ generated by the $X$ and $X^{\prime}$. Our theorem would be proved if we show that $X^{\prime \prime}$ has a dimension not greater than $d$, for then we must have $X=X^{\prime \prime}=X$ and hence $X$ must be defined over $K(u) \cap K\left(u^{\prime}\right)=K$. In the product space $U \times U \times A \times A \times A$ we consider the following subvarieties, where $y, y^{\prime}, y^{\prime \prime}$ are three independent generic points of $A$ over $K\left(u, u^{\prime}, x, x^{\prime}\right)$ : The locus $M$ of the point $u \times u^{\prime} \times x$ $\times x^{\prime} \times x+x^{\prime}$ over $K$, the locus $\Delta$ of the point $u \times u^{\prime} \times x \times y^{\prime} \times y^{\prime \prime}$ over $K$, the locus $\Delta^{\prime}$ of the point $u \times u^{\prime} \times y \times x^{\prime} \times y^{\prime \prime}$ over $K$, and the locus $\Gamma$ of the point $u \times u^{\prime} \times y \times y^{\prime} \times y+y^{\prime}$ over $K$. We have evidently the relations $M=\Delta \cap \Delta^{\prime} \cap \Gamma$ and $M \cap\left(u \times u^{\prime} \times A \times A \times A\right)=u \times u^{\prime} \times X \times X^{\prime} \times X^{\prime \prime}$. Let $N$ be the projection of $M$ into the partial product space $U \times U \times A$ of the first, second, and fifth factors, and consider the intersection $N \cap(u \times u \times A)$; since $N \cap(u \times u \times A)$ is evidently contained in the projection of $M \cap(u \times u \times A \times A \times A)$ into $U \times U \times A$, every point $u \times u \times x_{0}^{\prime \prime}$ in the former is the projection of a point $u \times u \times x_{0} \times x_{0}^{\prime} \times x_{0}^{\prime \prime}$ in the latter. The relation $u \times u \times x_{0} \times x_{0}^{\prime} \times x_{0}^{\prime \prime} \subset \Delta \cap \Delta^{\prime} \cap \Gamma$ implies that $x_{0} \subset X, x_{0}^{\prime} \subset X$, and $x_{0}^{\prime \prime}=x_{0}+x_{0}^{\prime}$, which shows that the point $x_{0}^{\prime \prime}$ is contained in $X$; it follows then that $N \cap(u \times u \times A)$ is contained in $u \times u \times X$ and hence has a dimension not greater than $d$. If $s$ is the dimension of $U$, then the dimension of $N$ cannot be greater than $2 s+d$, from which it follows that the dimension of $N \cap\left(u \times u^{\prime} \times A\right)=u \times u^{\prime} \times X^{\prime \prime}$ cannot be greater than $d$; this implies that the dimension of $X^{\prime \prime}$ cannot be greater than the dimension $d$ of $X$.

It might be not without some interest to indicate here an alternate proof of our theorem, which is simple but uses a deeper result of Weil. If $l$ is a positive integer which is prime to the characteristic of the field $K$, then the subgroup $g_{l}$ of all elements in $X$ whose orders divide $l$ is a finite group of order $l^{2 d}$, normally algebraic over $K(u)$ (Weil [6, Theorem 33, Corollary 1]). Since each point in $g_{l}$, being of finite order, is rational over $K$ ( $K$ being algebraically closed), the group $g_{l}$ is defined over $K$ and hence is normally algebraic over $K(u) \cap K=K$. It follows that for every point $x_{0}$ in $g_{l}$, the point $u^{\prime} \times x_{0}$ is a specialization of the point $u \times x_{0}$ over $K$, and since $u \times x_{0}$ is contained in 
$T, u^{\prime} \times x_{0}$ is also contained in $T$; this means that $g_{l}$ is contained in $X^{\prime}$, and hence is also contained in $X \cap X^{\prime}$. Since this is true for every positive integer $l$ prime to the characteristic of $K$, it follows from the same result quoted above that the dimension of the Abelian variety $X \cap X^{\prime}$ cannot be less than $d$ and hence must be equal to $d$. This means that $X=X^{\prime}$, and hence $X$ must be defined over $K(u) \cap K\left(u^{\prime}\right)=K$.

Theоrem 2. Let $A$ be an Abelian variety; let $K$ be a field of definition for $A$ and let $K^{*}$ be a primary extension of $K$. If an Abelian subvariety $X$ in $A$ is normally algebraic over $K^{*}$, then it is defined over $K$.

Proof. According to Theorem 1 , the Abelian variety $X$ is normally algebraic over $K^{*} \cap \bar{K}$ and hence is defined over a purely inseparable extension $K_{1}$ of $K^{*} \cap \bar{K}$. Since $K^{*} \cap \bar{K}$ is a primary extension of $K, K_{1}$ is also a purely inseparable extension of $K$; let $p^{e}$ be the degree of $K_{1}$ over $K$. Let $x$ be a generic point of $X$ over $K_{1}$, and denote by $x^{p^{0}}$ the point obtained from $x$ by raising all the coordinates of $x$ to the $p^{e}$ th power; since $K_{1}(x)$ is a regular extension of $K_{1}, K_{1}^{p^{e}}\left(x^{p^{c}}\right)$ is a regular extension of $K_{1}^{p^{e}}$, and since $K_{1}^{p^{e}}$ is contained in $K$, it follows that $K\left(x^{p^{\circ}}\right)$ is also a regular extension of $K$. Consider now the point $p^{e} x$ in $X$; since the associated point of 0 -cycle $p^{e}(x)$ in $A$ is rational over $K\left(x^{p^{e}}\right)$, the point $p^{e} x$ is rational over $K\left(x^{p^{e}}\right)$, i.e., we have $K\left(p^{e} x\right) \subset K\left(x^{p^{o}}\right)$ (Weil [6, Theorem 1], the proof holds for the more general situation considered here). This shows that $K\left(p^{e} x\right)$ is a regular extension of $K$. On the other hand, since $K_{1}(x)$ is a finite algebraic extension of $K_{1}\left(p^{e} x\right)$ (Weil [6, Proposition 24]), the point $p^{e} x$ is also a generic point of $X$ over $K_{1}$; and since $K\left(p^{e} x\right)$ is a regular extension of $K$ and $K_{1}$ is an algebraic extension of $K$, the point $p^{e} x$ has the same specializations over $K$ as it has over $K_{1}$. This proves the theorem.

REMARK. For any integer $e$ (positive or negative), the Abelian variety over $K^{p^{a}}$ defined by the point $x^{p^{e}}$ is called the $p^{e} t h$ power of the Abelian variety $A$.

Corollary 1. Let $A$ be an Abelian variety, defined over a field $K$, and let $K^{*}$ be a primary extension of $K$. If $B$ is an Abelian variety over $K^{*}$, and if $H$ is a rational homomorphism of $B$ into $A$, also defined over $K^{*}$, then the image $H(B)$ is an Abelian subvariety in $A$ which is defined over $K$. Furthermore, if the Abelian variety $B$ is also defined over $K$, then $H$ is defined over $K$.

Proof. The first part follows immediately from Theorem 2 and the fact that $H(B)$ is an Abelian variety defined over $K^{*}$. As to the second part, we observe that the graph of $H$ is an Abelian subvariety in the product variety $B \times A$, and since $H$ is defined over $K^{*}$ and $B \times A$ is defined over $K$, it follows from Theorem 2 that $H$ is also defined over $K$.

Corollary 2. Let $A$ be an Abelian variety, defined over a field $K$, and let $K^{*}=K(u)$ be a purely transcendental extension of $K$. If $B$ is an Abelian variety 
over $K^{*}$, and if $H$ is a rational transformation of $B$ into $A$, defined over $K^{*}$, then $H(B)$ is a subvariety in $A$ defined over $K$; furthermore, if $B$ is also defined over $K$, then $H$ is defined over $K$.

Proof. Since $H$ is defined over $K(u)$, the point $H(0)$ is rational over $K(u)$ and hence the correspondence $u \rightarrow H(0)$ defines a rational transformation of a projective space into $A$; it is well known that the image point $H(0)$ in this case is independent of $u$ and hence is rational over $K$. If $x$ is a generic point of $B$ over $K(u)$, then the correspondence $x \rightarrow H(x)-H(0)$ defines a rational homomorphism of $B$ into $A$, defined over $K(u)$, and the corollary then follows from Corollary 1.

3. An existence theorem. Let $K(u)$ be a regular extension of a field $K$, and let $A_{u}$ be an Abelian variety defined over $K(u)$; then, as we have explained in $\S 1$, the Abelian variety $A_{u}$ generates over $K$ an algebraic system of Abelian varieties. Let $u_{1}$ and $u_{2}$ be independent generic specializations of $u$ over $K$, and let $A_{1}$ and $A_{2}$ be respectively the specializations of $A_{u}$ over the specializations $u \rightarrow u_{1}$ and $u \rightarrow u_{2}$ over $K$; in general, the Abelian varieties $A_{1}$ and $A_{2}$ will not be isomorphic to each other. It is clear that if the "variable" Abelian variety $A_{u}$ is regularly isomorphic over $K(u)$ to a "fixed" Abelian variety defined over $K$, then $A_{1}$ and $A_{2}$ will be regularly isomorphic to each other over $K\left(u_{1}, u_{2}\right)$. We shall show in this section that the converse of this statement is also true; in other words, we shall show that if $A_{1}$ and $A_{2}$ are regularly isomorphic over $K\left(u_{1}, u_{2}\right)$, then there exists an Abelian variety $A$ defined over $K$, such that $A_{u}$ is regularly isomorphic to $A$ over $K(u)$ (Theorem 3 , Corollary $\left.1^{\prime}\right)$. However, for the purpose of application to the problem of the effect of ground field extension in the next section, we shall prove a somewhat more general result (Theorem 3 ), where the regular extension $K(u)$ is replaced by a separably generated extension. We shall need the following two lemmas.

Lemma 1. Let $K(u)$ be an extension of a field $K$, and let $V$ be a variety defined over $K(u)$; then, for almost every specialization $u \rightarrow u_{0}$ over $K$, the variety $V$ has a uniquely determined specialization $V_{0}$, which is a variety defined over $K\left(u_{0}\right)$.

Proof. Since the associated point $y$ of the variety $V$ is rational over $K(u)$, for almost every specialization $u \rightarrow u_{0}$ over $K$ the point $y$ has a uniquely determined specialization $y_{0}$ which is rational over $K\left(u_{0}\right)$, and hence the variety $V$ has a uniquely determined specialization $V_{0}$ which is a rational positive cycle over $K\left(u_{0}\right)$. Furthermore, it is well known that for almost every specialization $u \rightarrow u_{0}$ over $K$, the associated form of $V$, which is absolutely irreducible, will have a uniquely determined specialization which is also absolutely irreducible; one need only to observe that the condition of a form being absolutely reducible can be expressed as a system of algebraic equations in terms of the coefficients. The lemma then follows immediately. 
Lemma 2. Let $K(u)$ be an extension of a field $K$; let $V$ and $W$ be two varieties defined over $K(u)$, and let $F$ be a rational transformation of $V$ onto $W$, defined over $K(u)$, which is defined everywhere in $V$. Then, for almost every specialization $u \rightarrow u_{0}$ over $K$, there exist uniquely determined specializations $V_{0}, W_{0}, F_{0}$ of $V, W, F$ respectively, such that $V_{0}$ and $W_{0}$ are varieties defined over $K\left(u_{0}\right)$ and $F_{0}$ is a rational transformation of $V_{0}$ onto $W_{0}$, defined over $K\left(u_{0}\right)$.

Proof. That $V_{0}$ and $W_{0}$ are varieties defined over $K\left(u_{0}\right)$ follows from Lemma 1 ; furthermore, since $F$ is a subvariety in $V \times W$, it follows from Lemma 1 that $F_{0}$ is also a subvariety in $V_{0} \times W_{0}$, defined over $K\left(u_{0}\right)$. It remains to prove that the projection of $F_{0}$ onto $V_{0}$ is regular at every point of $V_{0}$. Let $S$ and $S^{\prime}$ be the ambient projective spaces of $V$ and $W$ respectively, and let $x$ be a generic point of $V$ over $K(u)$; without any loss of generality, we can assume that $K$ is algebraically closed, so that $u$ has a locus $U$ over $K$. Let $T$ be the locus of the point $u \times x \times F(x)$ over $K$ in $U \times S \times S^{\prime}$, and let $X$ be the locus of the point $u \times x$ over $K$ in $U \times S$; then we have the relations $T \cdot\left(u \times S \times S^{\prime}\right)=u \times F$ and $X \cdot(u \times S)=u \times V$ (Weil [5, Chap. VII, Theorem 12]). The projection of $T$ into $U \times S$ is $X$, and the projection of $T$ onto $X$ is regular; it can be shown that for almost every point $u_{0} \times x_{0}$ in $X$, the projection of $T$ onto $X$ is regular at $u_{0} \times x_{0}$. Since the projection of $T$ onto $X$ is regular at the point $u \times x^{\prime}$ for every point $x^{\prime}$ in $V$, it follows that for almost every point $u_{0}$ in $U$, the projection of $T$ onto $X$ is regular at the point $u_{0} \times x_{0}^{\prime}$ for every point $x_{0}^{\prime}$ in $V_{0}$. Since the projection of $T$ onto $X$ is regular at a point $u_{0} \times x_{0}$ if and only if the projection of $F_{0}$ onto $V_{0}$ is regular at $x_{0}$, we conclude that for almost every point $u_{0}$ in $U, F_{0}$ defines a rational transformation of $V_{0}$ on to $W_{0}$, defined everywhere in $V_{0}$.

THEOREM 3. Let $K^{*}$ be a primary extension of a field $K$, and let $K(v)$ be a separably generated extension of $K$, independent with respect to $K^{*}$ over $K$; let $L^{*}$ be an Abelian function field over $K^{*}$, independent with respect to $K^{*}(v)$ over $K^{*}$, and let $L$ be a subfield in $L^{*}(v)$ which is an Abelian function field over $K(v)$ and is independent with respect to $K^{*}(v)$ over $K(v)$. Let $v^{\prime}$ be a generic specialization of $v$ over $K$, independent with respect to $L^{*}(v)$ over $K$, and let $L^{\prime}$ be the subfield in $L^{*}\left(v^{\prime}\right)$ which is the image of $L$ under the isomorphism between $L^{*}(v)$ and $L^{*}\left(v^{\prime}\right)$. If for every choice of $v^{\prime}$ we have $L L^{\prime}=L\left(v^{\prime}\right)=L^{\prime}(v)$, then $L^{*} \cap L$ contains an Abelian function field $K(z)$ over $K$, independent with respect to $K(v)$ over $K$, such that $L=K(v, z)$.

REMARK. Since $K^{*}$ and $K(v)$ are linearly disjoint over $K, v^{\prime}$ is also a generic specialization of $v$ over $L^{*}$; hence $L^{*}(v)$ and $L^{*}\left(v^{\prime}\right)$ are isomorphic over $L^{*}$.

Proof. Let $A^{*}$ and $A$ be Abelian varieties over $K^{*}$ and $K(v)$ respectively defined by the fields $L^{*}$ and $L$ respectively, and let $F$ be the rational homomorphism of $A^{*}$ onto $A$, defined over $K^{*}(v)$, associated with the subfield $K^{*} L$ in $L^{*}(v)$; if $x$ is a generic point of $A^{*}$ over $K^{*}(v)$, then $y=F(x)$ is a generic point of $A$ over $K^{*}(v)$, and we can set $L^{*}=K^{*}(x)$ and $L=K(v, y)$. Without 
any loss of generality we can assume that both $A^{*}$ and $A$ are embedded in the same projective space $S_{n}$; furthermore, we can also assume that $K(v)$ is the defining field of $A$, for otherwise we can replace $A$ by the product of $A$ and the point $v$, and replace $S_{n}$ by another suitably chosen projective space. If $A^{\prime}$ and $F^{\prime}$ are respectively the specializations of $A$ and $F$ over the specialization $v \rightarrow v^{\prime}$ over $K^{*}$, then $A^{\prime}$ is an Abelian variety in $S_{n}$, with $K\left(v^{\prime}\right)$ as the defining field, and $F^{\prime}$ is a rational homomorphism of $A^{*}$ onto $A^{\prime}$ defined over $K^{*}\left(v^{\prime}\right)$; furthermore, $y^{\prime}=F^{\prime}(x)$ is a generic point of $A^{\prime}$ over $K^{*}\left(v^{\prime}\right)$, and we have $L^{\prime}=K\left(v^{\prime}, y^{\prime}\right)$. Let $v^{(1)}, \cdots, v^{(m)}$ be a set of independent generic specializations of $v^{\prime}$ over $K$, independent with respect to $L^{*}(v)$ over $K$, such that no two of them are specializations of each other over $K(v)$ and that every specialization of $v^{\prime}$ over $K$ is a specialization of one of these $m$ points over $K(v)$. For each $i=1, \cdots, m$, let $A^{(i)}$ and $F^{(i)}$ be respectively the specializations of $A^{\prime}$ and $F^{\prime}$ over the specialization $v^{\prime} \rightarrow v^{(i)}$ over $K^{*}$; then $A^{(i)}$ is an Abelian variety in $S_{n}$, with $K\left(v^{(i)}\right)$ as the defining field, and $F^{(i)}$ is a rational homomorphism of $A^{*}$ onto $A^{(i)}$, defined over $K^{*}\left(v^{(i)}\right)$. Furthermore, the point $y^{(i)}=F^{(i)}(x)$ is a generic point of $A^{(i)}$ over $K^{*}\left(v^{(i)}\right)$ and the field $L^{(i)}$ $=K\left(v^{(i)}, y^{(i)}\right)$ is the subfield in $L^{*}\left(v^{(i)}\right)$ which is the image of $L$ under the isomorphism between $L^{*}(v)$ and $L^{*}\left(v^{(i)}\right)$. We observe that the varieties $A^{(1)}, \cdots, A^{(m)}$ are all distinct. In fact, if we have $A^{(i)}=A^{(j)}$, then the field $K\left(v^{(i)}\right) \cap K\left(v^{(j)}\right)$ is a field of definition for both $A^{(i)}$ and $A^{(j)}$; we would then have $K\left(v^{(i)}\right)=K\left(v^{(i)}\right) \cap K\left(v^{(j)}\right)=K\left(v^{(j)}\right)$, which can only be possible for $i=j$. The condition $K\left(v, v^{(i)}, y, y^{(i)}\right)=L L^{(i)}=L\left(v^{(i)}\right)=K\left(v, v^{(i)}, y\right)$ is equivalent to the fact that there is a regular isomorphism $H^{(i)}$ of the Abelian variety $A$ onto the Abelian variety $A^{(i)}$, defined over $K\left(v, v^{(i)}\right)$, such that $y^{(i)}=H^{(i)}(y)$. We shall show presently (in order not to interrupt the argument now) that there exists a specialization $v^{\prime} \rightarrow v_{1}^{\prime}$ over $K$ with the following properties:

(1) The point $v_{1}^{\prime}$ is separably algebraic over $K$, and for each conjugate point $v_{i}^{\prime}(i=1, \cdots, d)$ of $v_{1}^{\prime}$ over $K$ there exists exactly one point $v^{\left(m_{i}\right)}$ among the points $v^{(1)}, \cdots, v^{(m)}$, such that $v_{i}^{\prime}$ is a specialization of $v^{\left(m_{i}\right)}$ over $K(v)$; we observe that the set $v_{1}^{\prime}, \cdots, v_{d}^{\prime}$ contains the complete set of conjugates of each $v_{i}^{\prime}$ over $K(v)$. (2) For each $i=1, \cdots, d$, there exist uniquely determined specializations $A^{\prime} \rightarrow A_{i}^{\prime}$ and $F^{\prime} \rightarrow F_{i}^{\prime}$ over the specialization $v^{\prime} \rightarrow v_{i}^{\prime}$ over $K^{*}$, such that $A_{i}^{\prime}$ is an Abelian variety over $K\left(v_{i}^{\prime}\right)$ and $F_{i}^{\prime}$ is a rational homomorphism of $A^{*}$ onto $A_{i}^{\prime}$, defined over $K^{*}\left(v_{i}^{\prime}\right)$; furthermore, the varieties $A_{1}^{\prime}, \cdots, A_{d}^{\prime}$ ' are all distinct subvarieties in $S_{n}$. We observe that for each $i=1, \cdots, d, A_{i}^{\prime}$ and $F_{i}^{\prime}$ are specializations of $A^{\left(m_{i}\right)}$ and $F^{\left(m_{i}\right)}$ respectively over the specialization $v^{\left(m_{i}\right)} \rightarrow v_{i}^{\prime}$ over $K^{*}(v)$. (3) For each $i=1, \cdots, d$, there exists a uniquely determined specialization $H_{i}$ of $H^{\left(m_{i}\right)}$ over the specialization $v^{\left(m_{i}\right)} \rightarrow v_{i}^{\prime}$ over $K(v)$, such that $H_{i}$ is a regular isomorphism of $A$ onto $A_{i}^{\prime}$, defined over $K\left(v, v_{i}^{\prime}\right)$, and we have the relation $H_{i}(y)=F_{i}^{\prime}(x)$. We observe that the set $H_{1}, \cdots, H_{d}$ contains the complete set of conjugates of each $H_{i}$ over $K(v)$. 
Consider now the set of $d$ distinct points $H_{1}(y), \cdots, H_{d}(y)$ in $S_{n}$; it determines a positive 0 cycle $Z(y)$ of degree $d$ in $S_{n}$, rational over $K(v, y)$. If $\Gamma$ is the canonical mapping of all positive 0 cycles of degree $d$ in $S_{n}$ into $M\left(S_{n} ; 0, d\right)$, then the mapping $y \rightarrow \Gamma(Z(y))$ defines a rational transformation $R$ of $A$ into $M\left(S_{n} ; 0, d\right)$, defined over $K(v)$. Let $V$ be the image variety $R(A)$ in $M\left(S_{n} ; 0, d\right)$, which is defined over $K(v)$; we maintain that $V$ is also defined over $K$. In fact, the relations $H_{i}(y)=F_{i}^{\prime}(x)$ show that the 0 -cycle $Z(y)$ is also rational over $K^{*}(x)$; it follows that the mapping $x \rightarrow \Gamma(Z(y))$ defines a rational transformation $R^{*}$ of $A^{*}$ into $M\left(S_{n} ; 0, d\right)$, defined over $K^{*}$, and since every specialization of $R(y)$ over $K(v)$ is a specialization of $R^{*}(x)$ over $K^{*}$ and viceversa, we have $R^{*}\left(A^{*}\right)=R(A)=V$, so that $V$ is defined over $K^{*}$ and hence also over $K(v) \cap K^{*}=K$.

Since the 0 -cycle $Z(y)$ consists of $d$ distinct points, each point $H_{i}(y)$ is separably algebraic over $K(z)$, where we set $z=R(y)$; and since furthermore $H_{i}(y)$ is the only point of $Z(y)$ contained in the variety $A_{i}^{\prime}$ and $y$ is the only point in $A$ with the image $H_{i}(y)$ in $A_{i}^{\prime}$ under the isomorphism $H_{i}$, it follows that $R$ is a birational transformation of $A$ onto $V$. Let $z_{0}$ be any point in $V$, and let $Z_{0}=\left(\zeta_{1}, \cdots, \zeta_{d}\right)$ be the positive 0 -cycles of degree $d$ determined by $z_{0}$; then each point $\zeta_{i}$ is in $A_{i}^{\prime}$ and $R^{-1}\left(z_{0}\right)$ must be one of the $d$ points $H_{i}^{-1}\left(\zeta_{i}\right)$ in $A$. This shows that $R$ has no fundamental points on $V$. Let $V_{1}$ be a derived normal model of the variety $V$ over $K$, and let $R_{1}$ be the birational transformation of $A$ onto $V_{1}$ induced by $R$; then $R_{1}^{-1}$ does not have any fundamental points on $V_{1}$. It follows then that $R_{1}^{-1}$ is defined everywhere on $V_{1}$; and since $R_{1}$ evidently has no fundamental points on $A$, it follows that $R_{1}$ is an everywhere biregular birational transformation of $A$ onto $V_{1}$. If $z_{1}$ and $z_{2}$ are two generic points of $V_{1}$ over $K$, we can define a composition function in $V_{1}$ by setting $z_{1}+z_{2}=R_{1}\left(R_{1}^{-1}\left(z_{1}\right)+R_{1}^{-1}\left(z_{2}\right)\right)$; it is easily seen that this composition function satisfies all the conditions of an Abelian variety. The function $z_{1}+z_{2}$ is evidently defined over $K(v)$; we maintain that this function is also defined over $K$. Let $R_{1}^{*}$ be the rational transformation of $A^{*}$ onto $V_{1}$ induced by $R^{*}$; it is clear that $R_{1}^{*}$ is defined over $K^{*}$, and the relation $R_{1}^{*}=R_{1} F$ shows that $R_{1}^{*}$ is a rational homomorphism of $A^{*}$ onto $V$, whereby $V$ is considered as an Abelian variety defined over $K(v)$. It follows that $R_{1}^{*-1}\left(z_{1}\right)$ and $R_{1}^{*-1}\left(z_{2}\right)$ are prime rational cycles in $A^{*}$ over $K^{*}\left(z_{1}, z_{2}\right)$; let $w_{1}$ and $w_{2}$ be generic points of $R_{1}^{*-1}\left(z_{1}\right)$ and $R_{1}^{*-1}\left(z_{2}\right)$ over $K^{*}\left(z_{1}, z_{2}\right)$ respectively. Then we have $z_{1}+z_{2}$ $=R_{1}^{*}\left(w_{1}+w_{2}\right)$, which shows that $z_{1}+z_{2}$ is rational over $K^{*}\left(z_{1}, z_{2}, w_{1}, w_{2}\right)$; and as $z_{1}+z_{2}$ is evidently independent of the choice of the generic points $w_{1}$ and $w_{2}$, it follows that $z_{1}+z_{2}$ is rational over a purely inseparable extension of $K^{*}\left(z_{1}, z_{2}\right)$. As $z_{1}+z_{2}$ is also rational over the separably generated extension $K\left(v, z_{1}, z_{2}\right)$ of $K\left(z_{1}, z_{2}\right)$, we conclude that $z_{1}+z_{2}$ is rational over $K\left(z_{1}, z_{2}\right)$. Thus we have shown that $V_{1}$ is an Abelian variety over $K$ and hence $K(z)$ is an Abelian function field over $K$; as we have evidently $L=K(v, z)$, this concludes 
the proof of our theorem, except for one point mentioned above, to which we shall now turn our attention.

In order to complete the proof of Theorem 3, we shall show the existence of a specialization $v_{1}^{\prime}$ of $v^{\prime}$ with the properties mentioned above. We observe first that the following properties hold for almost every specialization $v^{\prime} \rightarrow v_{0}^{\prime}$ over $K$ :

(1) There exists a unique specialization $A^{\prime} \rightarrow A_{0}^{\prime}$ such that $A_{0}^{\prime}$ is a nonsingular variety, defined over $K\left(v_{0}^{\prime}\right)$ (Lemma 1 );

(2) the composition function in $A^{\prime}$, defined everywhere on $A^{\prime} \times A^{\prime}$ to $A^{\prime}$, specializes uniquely into a function on $A_{0}^{\prime} \times A_{0}^{\prime}$ to $A_{0}^{\prime}$, defined over $K\left(v_{0}^{\prime}\right)$, which is defined everywhere; by the permanence of functional relations under specialization, this specialized composition function is also associative (Lemma 2);

(3) the unit element in $A^{\prime}$, being rational over $K\left(v^{\prime}\right)$, specializes uniquely into a point in $A_{0}^{\prime}$, rational over $K\left(v_{0}^{\prime}\right)$, which is also a unit for the specialized composition function, by the permanence of functional relations;

(4) the inverse function in $A^{\prime}$, defined everywhere in $A^{\prime}$, specializes uniquely into a function in $A_{0}^{\prime}$, defined over $K\left(v_{0}^{\prime}\right)$, which is also defined everywhere in $A_{0}^{\prime}$ (Lemma 2), and the permanence of functional relations shows that this function is also the inverse function in $A_{0}^{\prime}$ with respect to the specialized composition function and the specialized unit element;

(1)-(4) together shows that $A_{0}^{\prime}$ is an Abelian variety defined over $K\left(v_{0}^{\prime}\right)$;

(5) over the specialization $v^{\prime} \rightarrow v_{0}^{\prime}$ over $K^{*}$, the rational homomorphism $F^{\prime}$ of $A^{*}$ onto $A^{\prime}$ specializes uniquely into a rational transformation $F_{0}^{\prime}$ of $A^{*}$ onto $A_{0}^{\prime}$, defined over $K^{*}\left(v_{0}^{\prime}\right)$, which is defined everywhere in $A^{*}$ (Lemma 2 ); by the permanence of functional relations, this rational transformation $F_{0}^{\prime}$ is a homomorphism of $A^{*}$ onto $A_{0}^{\prime}$;

(6) if $v_{0}^{\prime}$ is a specialization of $v^{\left(m_{0}\right)}$ over $K(v)$, then there exists a uniquely determined specialization $H^{\left(m_{0}\right)} \rightarrow H_{0}$ such that $H_{0}$ is an everywhere biregular birational transformation of $A$ onto $A_{0}^{\prime}$, defined over $K\left(v, v_{0}^{\prime}\right)$, which by the permanence of functional relations must be an isomorphism of $A$ onto $A_{0}^{\prime}$ (Lemma 2, applied to both $H^{\left(m_{0}\right)}$ and its inverse); since we have the relation $H^{\left(m_{0}\right)}(y)=F^{\left(m_{0}\right)}(x)$, and since $F_{0}^{\prime}$ is also a specialization of $F^{\left(m_{0}\right)}$ over the specialization $v^{\left(m_{0}\right)} \rightarrow v_{0}^{\prime}$ over $K^{*}(v)$ and $H_{0}$ is also a specialization of $H^{\left(m_{0}\right)}$ over the specialization $v^{\left(m_{0}\right)} \rightarrow v_{0}^{\prime}$ over $K^{*}(v)$, it follows that $H_{0}(y)=F_{0}^{\prime}(x)$.

Now, let $w_{1}, \cdots, w_{r}$ be a separating transcendental base of $K\left(v^{\prime}\right)$; then the following properties hold for almost every specialization $w \rightarrow w_{0}$ over $K$ :

(7) there are only a finite number of specializations $v_{1}^{\prime}, \cdots, v_{d}^{\prime}$ of $v^{\prime}$ over the specialization $w \rightarrow w_{0}$ over $K$, forming a complete set of conjugates over $K\left(w_{0}\right)$;

(8) each point $v_{i}^{\prime}$ is separably algebraic over $K\left(w_{0}\right)$; 
(9) each point $v_{i}^{\prime}$ is the specialization of exactly one point $v^{\left(m_{i}\right)}$ over $K(v)$; (10) each point $v_{i}^{\prime}$ satisfies the conditions (1)-(6) above.

The proof of (7), (8), (10) follows immediately from the fact that each is expressed by the condition that a system of algebraic equations over $K$ is not satisfied by the point $w_{0}$ or the point $v_{i}^{\prime}$.

Thus we have shown that almost every specialization $v_{1}^{\prime}$ of $v^{\prime}$ over $K$ has all the desired properties except the condition that $v_{1}^{\prime}$ be separably algebraic over $K$. For this last condition, it is clearly sufficient to show that there exists at least one point $w_{0}$ in the $r$-space which is separably algebraic over $K$ and is not contained in a given bunch of varieties, and this follows immediately from the fact that the set of all separably algebraic points over $K$ in the $r$ space is everywhere dense with respect to the Zariski topology (in which the closed subsets are the bunches of subvarieties). In fact, this is obvious in case of the 1-space, there being more than a finite number of separably algebraic elements over $K$ in the universal domain, and the general case can be easily deduced from this by induction.

Corollary 1. Let $K(v)$ be a regular extension of $K$, and let $L$ be an Abelian function field over $K(v)$; let $v^{\prime}$ be a generic specialization of $v$ over $K$, independent with respect to $v$ over $K$, and let $L^{\prime}$ be an Abelian function field over $K\left(v^{\prime}\right)$ such that the isomorphism between $K(v)$ and $K\left(v^{\prime}\right)$ can be extended to an isomorphism between $L$ and $L^{\prime}$. If the field $L^{\prime}$ can be so chosen that $L L^{\prime}=L\left(v^{\prime}\right)=L^{\prime}(v)$, then $L$ contains an Abelian function field $K(z)$ over $K$, independent with respect to $K(v)$ over $K$, such that $L=K(v, z)$.

Proof. The corollary follows from Theorem 3 if we take $u$ to be a generic specialization of $v$ over $K$, independent with respect to $L$ over $K$, and set $K^{*}=K(u)$ and take $L^{*}$ to be an Abelian function field over $K(u)$ such that the isomorphism between $K(v)$ and $K(u)$ can be extended to an isomorphism of $L$ and $L^{*}$ and that the relation $L L^{*}=L^{*}(v)=L(u)$ holds; for we would then have the relation $L^{*}\left(v, v^{\prime}\right)=L^{*} L\left(v^{\prime}\right)=L\left(u, v^{\prime}\right)=L^{\prime}(u, v)$, from which it follows by Theorem 2, Corollary 1, that $L^{*}\left(v^{\prime}\right)=L^{\prime}(u)$. One observes that the condition "for any choice of $v^{\prime}$ " is not necessary here, for all such $v^{\prime}$ are generic specializations of each other over $K(v)$.

In order to exhibit the geometrical meaning of this corollary and also for the sake of convenience in later applications, we shall restate it in terms of Abelian varieties instead of function fields:

Corollary $1^{\prime}$. Let $K(v)$ be a regular extension of $K$, and let $A$ be an Abelian variety defined over $K(v)$; let $v^{\prime}$ be a generic specialization of $v$ over $K$, independent with respect to $v$ over $K$, and let $A^{\prime}$ be the specialization of $A$ over the specialization $v \rightarrow v^{\prime}$ over $K$. If $A$ is regularly isomorphic to $A^{\prime}$ over $K\left(v, v^{\prime}\right)$, then $A$ is regularly isomorphic to an Abelian variety defined over $K$.

COROllary 2. Let $V$ be a variety defined over a field $K$, and let $K(u)$ be a 
regular extension of $K$. If $V$ is birationally equivalent over $K(u)$ to an Abelian variety defined over $K(u)$, then $V$ is also birationally equivalent over $K(u)$ to an $A$ belian variety $A$ defined over $K$; furthermore, this Abelian variety $A$ is uniquely determined up to a regular isomorphism over $K$, independent of the choice of the regular extension $K(u)$ of $K$.

Proof. Let $u^{\prime}$ be a generic specialization of $u$ over $K$, independent of $u$ over $K$, and let $x$ be a generic point of $V$ over $K\left(u, u^{\prime}\right)$; then $K(u, x)$ is an Abelian function field over $K(u)$ and $K\left(u^{\prime}, x\right)$ is an Abelian function field over $K\left(u^{\prime}\right)$, and since $K\left(u, x, u^{\prime}\right)=K\left(u^{\prime}, x, u\right)$, it follows from Corollary 1 that $K(u, x)=K(u, z)$, where $K(z)$ is an Abelian function field over $K$, independent with respect to $K(u)$ over $K$. If $A$ is the Abelian variety over $K$, determined by $K(z)$, then $V$ is evidently birationally equivalent to $A$ (over $K(u)$ ); if $A_{1}$ is an Abelian variety over $K$ obtained in this manner with respect to another regular extension $K_{1}$ of $K$, then $A_{1}$ must also be birationally equivalent to $V$ over $K_{1}$ and hence must be regularly isomorphic to $A$ over $K_{1}(u)$; according to Theorem 2, Corollary 1 , then $A_{1}$ must be regularly isomorphic to $A$ over $K$.

4. The $K$-image.

THEOREM 4. Let $K^{*}$ be a primary extension of $K$, and let $A^{*}$ be an Abelian variety over $K^{*}$. Then there exists an Abelian variety $A$ over $K$, uniquely determined up to a regular isomorphism over $K$, and a rational homomorphism $F$ of $A^{*}$ onto $A$, defined over $K^{*}$, such that if $H$ is a rational homomorphism of $A^{*}$ into an Abelian variety $B$ and if $B$ is defined over a separably generated extension $K(v)$ of $K$, independent with respect to $K^{*}$ over $K$, and $H$ is defined over $K^{*}(v)$, then $H$ is the product of $F$ and a rational homomorphism of $A$ into $B$, defined over $K(v)$.

Proof. Let $x$ be a generic point of $A^{*}$ over $K^{*}$, and set $L^{*}=K^{*}(x)$; if $F_{0}$ is any rational homomorphism of $A^{*}$ onto an Abelian variety $A_{0}$ defined over $K$ and if $F_{0}$ is defined over $K^{*}$, then $L_{0}=K\left(F_{0}(x)\right)$ is an Abelian function field over $K$ which is a subfield in $L^{*}$ and is linearly disjoint with respect to $K^{*}$ over $K$. This field $L_{0}$, being the function field of $A_{0}$ over $K$, determines $A_{0}$ uniquely up to a regular isomorphism over $K$, and $F_{0}$ is the rational homomorphism associated with the subfield $K^{*} L_{0}$ in $L^{*}$ and hence is uniquely determined up to regular isomorphisms of $A^{*}$ and $A_{0}$ over $K^{*}$ and $K$ respectively. Consider now the aggregate $\mathfrak{R}$ of all such subfields $K\left(F_{0}(x)\right)$ in $L^{*}$ as $F_{0}$ runs through all such homomorphisms; let $L_{1}$ and $L_{2}$ be two fields in $\&$, and let $F_{1}$ and $F_{2}$ be the corresponding rational homomorphisms of $A^{*}$ onto the Abelian varieties $A_{1}$ and $A_{2}$ respectively, so that we have $L_{1}=K\left(F_{1}(x)\right)$ and $L_{2}=K\left(F_{2}(x)\right)$. The rational transformation $F_{1} \times F_{2}$ is a homomorphism of $A^{*}$ into the product Abelian variety $A_{1} \times A_{2}$, and the image of $A^{*}$ in $A_{1} \times A_{2}$ under this homomorphism is then an Abelian subvariety $A_{3}$, defined over $K^{*}$; since $A_{1} \times A_{2}$ is defined over $K$, it follows from Theorem 2, Corollary 
1 , that the Abelian variety $A_{3}$ is defined not only over $K^{*}$, but also over $K$.

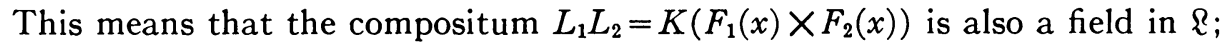
it follows then from [2, Lemma 1$]$ that there is a maximal field $L$ in $\mathfrak{R}$, and we can define $A$ to be the Abelian variety over $K$ defined by $L$ and define $F$ to be a homomorphism of $A^{*}$ onto $A$ associated with the subfield $L$ of $L^{*}$. Consider now the homomorphism $H$ and the extension $K^{*}(v)$ of $K$ over which $H$ is defined; then the last assertion of our theorem is equivalent to the statement that if we replace the fields $K$ and $K^{*}$ by $K(v)$ and $K^{*}(v)$ respectively, and if we denote by $\mathfrak{l}_{v}$ the aggregate of all subfields in $L^{*}(v)$ of the form $K\left(v, F_{0}(x)\right)$, where $F_{0}$ is any rational homomorphism, defined over $K^{*}(v)$, of $A^{*}$ onto an Abelian variety defined over $K(v)$, then the maximal field $L_{v}$ in $\mathfrak{R}_{v}$ is the field $L(v)$. To show this, we consider a field $K_{1}$ which is the separably-algebraic closure of an infinite transcendental extension of $K$; it is well known (MacLane [4, Theorem 9(a) and Theorem 15]) that any finitely generated extension of $K$ in $K_{1}$ is a separably generated extension of $K$, and it is easily seen that for any finitely and separably generated extension of $K$ there exists an isomorphic extension of $K$ in $K_{1}$ which is independent with respect to it over $K$. If we now apply the preceding argument with $K_{1}$ replacing $K$ as the ground field and if $A$ is the so obtained Abelian variety over $K_{1}$ and $F$ is a homomorphism of $A^{*}$ onto $A$ associated with it, then there is a finitely and separably generated extension of $K$ in $K_{1}$ over which both $A$ and $F$ are defined. It is clearly sufficient to prove our assertion for the case where $K(v)$ is such a field, so that $A$ is the Abelian variety over $K(v)$ defined by $L_{v}$. Let $v^{\prime}$ be a generic specialization of $v$ over $K$ such that $K\left(v^{\prime}\right)$ is contained in $K_{1}$ and is independent with respect to $K(v)$ over $K$, and let $L_{v^{\prime}}$ be the maximal field in the aggregate $\mathfrak{R}_{v^{\prime}}$, where $\mathfrak{R}_{v^{\prime}}$ is defined similarly as $\mathfrak{R}_{v}$ with $K\left(v^{\prime}\right)$ replacing $K(v)$; similarly, let $L_{\left(v, v^{\prime}\right)}$ be the maximal field in the aggregate $\mathfrak{R}_{\left(v, v^{\prime}\right)}$. Then it follows from the definition of $A$ that we have the relation $L_{\left(v, v^{\prime}\right)}=L_{v}\left(v^{\prime}\right)$, and since $L_{\left(v, v^{\prime}\right)} \supset L_{v} L_{v^{\prime}} \supset L_{v}\left(v^{\prime}\right)$, we have the relation $L_{v} L_{r^{\prime}}=L_{v}\left(v^{\prime}\right)$. It follows then from Theorem 3 that $L^{*} \cap L_{v}$ contains an Abelian function field $K(z)$ over $K$, independent with respect to $K(v)$ over $K$, such that $L_{v}=K(z, v)$; since $K(z)$ is evidently a field in $\mathfrak{R}$, we have the relation $L \supset K(z)$ and hence $L(v) \supset K(z, v)=L_{v}$, which shows that $L(v)=L_{v}$. This concludes the proof of our theorem.

The Abelian variety $A$ in Theorem 4 is called the $K$-image of $A^{*}$ over $K^{*}$, and the rational homomorphism $F$ is called the canonical homomorphism of $A^{*}$ onto $A$. The $K$-image $A$ of $A^{*}$ over $K^{*}$ is uniquely determined up to a regular isomorphism over $K$, and the canonical homomorphism $F$ of $A^{*}$ onto $A$ is uniquely determined up to regular isomorphisms of $A^{*}$ and $A$ over $K^{*}$ and $K$ respectively. If $K_{1}$ is any separably generated extension of $K$, independent with respect to $K^{*}$ over $K$, then the $K$-image $A$ of $A^{*}$ over $K^{*}$ is also the $K_{1}$-image of $A^{*}$ over $K^{*} K_{1}$, with the same canonical homomorphism $F$; furthermore, it is easily seen from Theorem 2, Corollary 1 , and the proof of 
Theorem 4 that if $K_{1}^{*}$ is any primary extension of $K^{*}$, then the $K$-image $A$ of $A^{*}$ over $K^{*}$ is also the $K$-image of $A^{*}$ over $K_{1}^{*}$, with the same canonical homomorphism $F$.

CoRollary. Let $K_{1}$ be any extension of $K$, independent with respect to $K^{*}$ over $K$, and let $A_{1}$ be the $K_{1}$-image of $A^{*}$ over $K_{1} K^{*}$ and $F_{1}$ be the canonical homomorphism of $A^{*}$ onto $A_{1}$; then there is a rational isomorphism $I$ of $A_{1}$ onto $A$, defined over $K_{1}$, such that $F=I F_{1}$.

Proof. Without any loss of generality, we can assume that $K_{1}$ is a purely inseparable extension of $K$, for otherwise we can replace $K$ by its separablyalgebraic closure in $K_{1}$, and $A$ would still remain the $K$-image of $A^{*}$. Let $F_{1}$ be the canonical homomorphism of $A^{*}$ onto $A_{1}$; then according to Theorem $4, F$ is the product of $F_{1}$ and a rational homomorphism $I$ of $A_{1}$ onto $A$, defined over $K_{1}$. It follows then that $A$ is also the $K$-image of $A_{1}$ over $K_{1}$, and $I$ is the corresponding canonical homomorphism. Since $K_{1}$ is purely inseparable over $K$, there exists a positive integer $e$ such that the $p^{\circ}$ th power $A_{2}$ of $A_{1}$ is an Abelian variety over $K$ and there is a rational isomorphism $I_{1}$ of $A_{1}$ onto $A_{2}$, defined over $K_{1}$. According to Theorem 4, $I_{1}$ is the product of $I$ and a rational homomorphism of $A$ onto $A_{2}$; this shows that $I$ must be an isomorphism.

Theorem 4 enables us to obtain a stronger form of Theorem 2 in our note [2], where the hypothesis "if both $B$ and $H$ are defined over $K$ " is replaced by "if both $B$ and $H$ are defined over a separably generated extension of $K$." We shall state this stronger result here, as a theorem, mainly on account of its own interest, although we shall also find it convenient to use it in the proof of the next theorem.

Theorem 5. Let $A$ be an Abelian variety over a field $K$, and let $X$ be an algebraic subgroup in $A$, normally algebraic over $K$; let $A(X, K)$ be the quotient Abelian variety of $A$ over $X$ relative to $K$, and let $F$ be the canonical homomorphism of $A$ onto $A(X, K)$. If $H$ is any rational homomorphism of $A$ into an Abelian variety $B$ with $X$ as the kernel and if both $B$ and $H$ are defined over a separably generated extension $K(v)$ of $K$, then $H$ is the product of $F$ and $a$ rational isomorphism of $A(X, K)$ into $B$, defined over $K(v)$.

Proof. Since $X$ is normally algebraic over $K$, there is a purely inseparable extension $K_{1}$ of $K$ such that $X$ is normally algebraic and separable over $K_{1}$; according to [2, Theorem 1$]$, the quotient variety $A(X)$ and the canonical homomorphism $F_{1}$ of $A$ onto $A(X)$ are both defined over $K_{1}$. Let $A(X, K)$ be the $K$-image of $A(X)$ over $K_{1}$ and $F_{2}$ be the canonical homomorphism of $A(X)$ onto $A(X, K)$, and set $F=F_{2} F_{1}$; since both $A$ and $A(X, K)$ are defined over $K$, it follows from Theorem 2, Corollary 1 , that $F$ is also defined over $K$. It is then easily seen from Theorem 4 and [2, Theorem 1], that the so defined $A(X, K)$ and $F$ have the property stated in the theorem.

Theorem 6. Let $K^{*}$ be a primary extension of $K$, and let $A^{*}$ be an Abelian 
variety over $K^{*}$; let $K_{1}$ be any extension of $K$, independent with respect to $K^{*}$ over $K$. If there is a rational homomorphism $H$ of an Abelian variety $B$ onto $A^{*}$, and if $B$ is defined over $K_{1}$ and $H$ is defined over $K^{*} K_{1}$, then the canonical homomorphism $F$ of $A^{*}$ onto its own $K$-image $A$ over $K^{*}$ is an isomorphism.

Proof. Without any loss of generality, we can assume that $K$ is algebraically closed and $K_{1}=K$; for otherwise we can replace $K$ by $\bar{K}_{1}$ and $K^{*}$ by $K^{*} \bar{K}_{1}$, and if the canonical homomorphism $F_{1}$ of $A^{*}$ onto its $\bar{K}_{1}$-image $A_{1}$ over $K^{*} \bar{K}_{1}$ is an isomorphism, then according to Theorem 4, Corollary, the canonical homomorphism $F$ is the product of $F_{1}$ and a rational isomorphism of $A_{1}$ onto $A$, and hence is also an isomorphism.

Let $X$ be the kernel of $H$ in $B$; since $X$ is normally algebraic over $K^{*}$ and since $B$ is defined over $K$, it follows from Theorem 1 that $X$ is also normally algebraic over $K$. According to Theorem $5, H$ is the product of the canonical homomorphism of $B$ onto $B(X, K)$ and a rational isomorphism $R$ of $B(X, K)$ on to $A^{*}$, defined over $K^{*}$. Let $x$ be a generic point of $B(X, K)$ over $K$; then $y=R(x)$ is a generic point of $A^{*}$ over $K^{*}$ and the field $K^{*}(x)$ is a purely inseparable extension of $K^{*}(y)$. Let $e$ be the exponent of $K^{*}(x)$ over $K^{*}(y)$, and consider the $p^{e}$ th power $A_{2}$ of $B(X, K)$, whose function field over $K^{*}$ is isomorphic to $K^{*}\left(x^{p^{b}}\right)$, where $x^{p^{b}}$ denotes the point obtained from $x$ by raising all coordinates of $x$ to the $p^{e}$ th power. Since we have $K^{*}(x) \supset K^{*}(y) \supset K^{*}\left(x^{p^{e}}\right)$, $K^{*}(y)$ is evidently a purely inseparable extension of $K^{*}\left(x^{p^{e}}\right)$; it follows that there is a rational isomorphism of $A^{*}$ onto $A_{2}$, which according to Theorem 4 must be a product of $F$ and a rational homomorphism of $A$ onto $A_{2}$. This shows that $F$ must be an isomorphism.

REMARK. It is easily seen that the condition in Theorem 6 is not only sufficient but also necessary.

Corollary 1. Let $K^{*}$ be a primary extension of $K$, and let $A^{*}$ be an Abelian variety over $K^{*}$; if the canonical homomorphism $F$ of $A^{*}$ onto its $K$-image $A$ over $K^{*}$ has a finite kernel, then $F$ is an isomorphism.

Proof. According to Weil [6, Theorem 27], there is a rational homomorphism of $A$ onto $A^{*}$, defined over $K^{*}$, and the corollary then follows from Theorem 6.

Corollary 2. Let $K^{*}$ be a primary extension of $K$, and let $A^{*}$ be an Abelian variety over $K^{*}$; then the kernel of the canonical homomorphism $F$ of $A^{*}$ onto its $K$-image $A$ over $K^{*}$ is an Abelian subvariety in $A^{*}$.

Proof. The kernel $X$ of $F$ is an algebraic subgroup in $A^{*}$, normally algebraic over $K^{*}$; let $X_{0}$ be the maximal Abelian variety contained in $X$, which is also normally algebraic over $K^{*}$, and consider the quotient variety $A^{*}\left(X_{0}, K^{*}\right)$ and the canonical homomorphism $F^{*}$ of $A^{*}$ onto $A^{*}\left(X_{0}, K^{*}\right)$, both defined over $K^{*}$. According to Theorem $5, F$ is the product of $F^{*}$ and a 
rational homomorphism $H$ of $A^{*}\left(X_{0}, K^{*}\right)$ onto $A$, defined over $K^{*}$, and it is easily seen that $A$ is also the $K$-image of $A^{*}\left(X_{0}, K^{*}\right)$ over $K^{*}$ and $H$ is the canonical homomorphism. Since the kernel of $H$ is isomorphic to the quotient group $X / X_{0}$ and hence is finite, it follows from Corollary 1 that $H$ is an isomorphism; this shows that $X=X_{0}$.

\section{The $K$-trace.}

Theorem 7. Let $K^{*}$ be a primary extension of $K$, and let $A^{*}$ be an Abelian variety over $K^{*}$. Then there exists a uniquely determined Abelian subvariety $A_{0}{ }^{*}$ in $A^{*}$, defined over $K^{*}$, with the properties: (1) If $H$ is a rational homomorphism of an Abelian variety $B$ into $A^{*}$, and if $B$ is defined over any extension $K(v)$ of $K$, independent with respect to $K^{*}$ over $K$, and $H$ is defined over $K^{*}(v)$, then $H(B)$ is contained in $A_{0}{ }^{*}$, (2) $A_{0}^{*}$ is the smallest Abelian subvariety in $A^{*}$ which has this property. Furthermore, $A_{0}{ }^{*}$ is the only Abelian subvariety in $A^{*}$ which is isogenous to the $K$-image of $A^{*}$ over $K^{*}$, and the canonical homomorphism $F_{0}$ of $A_{0}{ }^{*}$ onto its own $K$-image $A_{0}$ over $K^{*}$ is an isomorphism.

Proof. Let $K_{1}$ and $K_{2}$ be two extensions of $K$ which are both independent with respect to $K^{*}$ over $K$, and let $B_{1}$ and $B_{2}$ be Abelian varieties defined over $K_{1}$ and $K_{2}$ respectively; let $H_{1}$ and $H_{2}$ be rational homomorphisms of $B_{1}$ and $B_{2}$ respectively into $A^{*}$, and let $A_{1}{ }^{*}$ and $A_{2}{ }^{*}$ be the respective image varieties in $A^{*}$. Let $K^{* \prime}$ be an extension of $K^{*}$ which is isomorphic to $K^{*} K_{2}$ over $K$ and is independent with respect to $K^{*} K_{1}$ over $K^{*}$, and let $K_{2}^{\prime}$ be the subfield in $K^{* \prime}$ which is the image of $K_{2}$ under the isomorphism between $K^{*} K_{2}$ and $K^{* \prime}$; then $K_{2}^{\prime}$ is independent with respect to $K^{*} K_{1}$ over $K$, and hence $K_{2}^{\prime} K_{1}$ is independent with respect to $K^{*}$ over $K$, and we have the relation $K^{*} K_{2}^{\prime}$ $=K^{* \prime}$. Let $B_{2}^{\prime}$ be the Abelian variety defined over $K_{2}^{\prime}$ which is the isomorphic image of $B_{2}$ under the isomorphism between $K_{2}$ and $K_{2}^{\prime}$ over $K$; since $K^{*} K_{2}$ is isomorphic to $K^{*} K_{2}^{\prime}$ over $K^{*}$, there is a rational homomorphism $H_{2}^{\prime}$ of $B_{2}^{\prime}$ into $A^{*}$ such that $H_{2}^{\prime}\left(B_{2}^{\prime}\right)=H_{2}\left(B_{2}\right)=A_{2}{ }^{*}$. The product Abelian variety $B_{1} \times B_{2}^{\prime}$ is then defined over $K_{1} K_{2}^{\prime}$, which is independent with respect to $K^{*}$ over $K$, and $H_{1} p r_{1}+H_{2} p r_{2}$ is a rational homomorphism of $B_{1} \times B_{2}^{\prime}$ into $A^{*}$ which has as its image the Abelian subvariety in $A^{*}$ generated by $A_{1}{ }^{*}$ and $A_{2}{ }^{*}$. Consider now the aggregate of all Abelian subvarieties in $A^{*}$ which are rationally homomorphic images of Abelian varieties defined over fields which are independent with respect to $K^{*}$ over $K$; it follows from what we have just said that there is an Abelian subvariety $A_{0}{ }^{*}$ in this aggregate which contains every variety in the aggregate. It is clear that $A_{0}{ }^{*}$ has the properties (1) and (2); furthermore, it follows from Theorem 6 that $F_{0}$ is an isomorphism of $A_{0}{ }^{*}$ onto $A_{0}$. Consider now the $K$-image $A$ of $A^{*}$ over $K^{*}$; according to the Poincaré Complete Reducibility Theorem (Weil [6, Theorem 26 and Corollary 2]), there exists a homomorphism $H$ of $A$ onto an Abelian subvariety of the same dimension in $A^{*}$, and it can be easily seen from the cited proof of this theorem that $H$ is defined over $K^{*}$. It follows that $H(A)$ is contained in $A_{0}{ }^{*}$, so 
that the dimension of $A_{0}{ }^{*}$ is not less than that of $A$. On the other hand, by the same theorem, there exists a homomorphism $H_{1}$ of $A^{*}$ onto $A_{0}{ }^{*}$, defined over $K^{*}$; then $F_{0} H_{1}$ is a homomorphism of $A^{*}$ onto $A_{0}$, defined over $K^{*}$, and since $F_{0} H_{1}$ is according to Theorem 4 the product of the canonical homomorphism $F$ of $A^{*}$ onto $A$ and a homomorphism of $A$ onto $A_{0}$, it follows that the dimension of $A_{0}$ and hence also the dimension of $A_{0}{ }^{*}$ cannot be greater than that of $A$. Thus $H(A)$ and $A_{0}^{*}$ have the same dimension and hence we have $H(A)=A_{0}{ }^{*}$, so that $A$ and $A_{0}{ }^{*}$ are isogenous over $K^{*}$ (and hence $A$ and $A_{0}$ are isogenous over $K$ ); and it is clear from our argument that the Abelian subvariety $A_{0}{ }^{*}$ is the only Abelian subvariety in $A^{*}$ which is isogenous to the Abelian variety $A$.

The Abelian variety $A_{0}{ }^{*}$ in Theorem 7 is called the $K$-maximal Abelian subvariety over $K^{*}$ in $A^{*}$; it is uniquely determined by the Abelian variety $A^{*}$ and the fields $K^{*}$ and $K$. If $K_{1}$ is any extension of $K$, independent with respect to $K^{*}$ over $K$, then the $K$-maximal Abelian subvariety $A_{0}{ }^{*}$ over $K^{*}$ in $A^{*}$ is also the $K_{1}$-maximal Abelian subvariety over $K^{*} K_{1}$ in $A^{*}$; and if $K_{1}^{*}$ is any primary extension of $K^{*}$, then the $K$-maximal Abelian subvariety $A_{0}{ }^{*}$ over $K^{*}$ in $A^{*}$ is also the $K$-maximal Abelian subvariety over $K_{1}^{*}$ in $A^{*}$.

Theorem 8. Let $K^{*}$ be a regular extension of $K$ and let $A^{*}$ be an Abelian variety over $K^{*}$. Then there exists an Abelian variety $A^{\prime}$ over $K$, uniquely determined up to a regular isomorphism over $K$, and a rational isomorphism $F$ of $A^{\prime}$ into $A^{*}$, defined over $K^{*}$, such that if $B$ is an Abelian variety defined over any extension $K_{1}$ of $K$, independent with respect to $K^{*}$ over $K$, and if $H$ is a rational homomorphism of $B$ into $A^{*}$, defined over $K^{*} K_{1}$, then $H$ is the product of $F$ and a rational homomorphism of $B$ into $A^{\prime}$, defined over $K_{1}$.

Proof. Without any loss of generality, we can assume that $K^{*}=K(u)$ is a finitely generated extension of $K$; let $A$ be the $K$-image of $A^{*}$ over $K^{*}$ and $A_{u}$ be the maximal $K$-Abelian subvariety over $K^{*}$ in $A^{*}$, and let $T_{u}$ be a rational homomorphism of $A$ onto $A_{u}$, defined over $K(u)$. Let $u_{1}(=u), u_{2}, \cdots, u_{m}$ be $m$ independent generic specializations of $u$ over $K$; if $S_{n}$ is the ambient projective space of $A_{u}$, then there exist Abelian varieties $A_{u_{1}}, \cdots, A_{u_{m}}$ in $S_{n}$ which are respectively the specializations of $A_{u}$ over the specializations $u_{1}, \cdots, u_{m}$ of $u$ over $K$. If $y$ is a generic point of $A$ over $K\left(u_{1}, \cdots, u_{m}\right)$, then for each $i=1, \cdots, m$, the point $x_{i}=T_{u_{i}}(y)$ is a generic point of $A_{u_{i}}$ over $K\left(u_{1}, \cdots, u_{m}\right)$. The correspondence $y \rightarrow\left(x_{1}, \cdots, x_{m}\right)$ then defines a rational homomorphism $T_{m}$ of $A$ into the product variety $A_{u_{1}} \times \cdots \times A_{u_{m}}$, defined over $K\left(u_{1}, \cdots, u_{m}\right)$, and the image $T_{m}(A)$ is an Abelian variety, defined over $K\left(u_{1}, \cdots, u_{m}\right)$, whose projection into the first factor $A_{u_{1}}=A_{u}$ is a rational isomorphism onto $A_{u}$, i.e., $\operatorname{pr}_{1}\left(T_{m}(A)\right)=s A_{u}$, where $s$ is the degree of $K\left(u_{1}, \cdots, u_{m}, x_{1}, \cdots, x_{m}\right)$ over $K\left(u_{1}, \cdots, u_{m}, x_{1}\right)$.

Since $K\left(u_{1}, \cdots, u_{m}, y\right)$ is a finite extension of $K\left(u_{1}, \cdots, u_{m}, x_{1}\right)$ whose degree is independent of the integer $m$, and since we have $K\left(u_{1}, \cdots, u_{m}, y\right)$ 
$\supset K\left(u_{1}, \cdots, u_{m}, x_{1}, \cdots, x_{m}\right) \supset K\left(u_{1}, \cdots, u_{m}, x_{1}\right)$, it follows that the degree $s$ of $K\left(u_{1}, \cdots, u_{m}, x_{1}, \cdots, x_{m}\right)$ over $K\left(u_{1}, \cdots, u_{m}, x_{1}\right)$ is bounded for all $m$. We shall assume that the integer $m$ has been chosen so large that this degree reaches the maximum; let $u_{1}^{\prime}, \cdots, u_{m}^{\prime}$ be $m$ independent generic specializations of $u$ over $K$, independent with respect to $u_{1}, \cdots, u_{m}$, and $y$ over $K$, and set $x_{i}^{\prime}=T_{u_{i^{\prime}}}(y), i=1, \cdots, m$. Since $K\left(u_{1}, \cdots, u_{m}, u_{1}^{\prime}, \cdots, u_{m}^{\prime}\right.$, $\left.x_{1}, \cdots, x_{m}, x_{1}^{\prime}, \cdots, x_{m}^{\prime}\right)$ contains $K\left(u_{1}, \cdots, u_{m}, u_{1}^{\prime}, \cdots, u_{m}^{\prime}, x_{1}, \cdots, x_{m}\right)$ and has the same degree $s$ over $K\left(u_{1}, \cdots, u_{m}, u_{1}^{\prime}, \cdots, u_{m}^{\prime}, x_{1}\right)$ as the latter, we must have the relation $K\left(u_{1}, \cdots, u_{m}, u_{1}^{\prime}, \cdots, u_{m}^{\prime}, x_{1}, \cdots, x_{m}\right.$, $\left.x_{1}^{\prime}, \cdots, x_{m}^{\prime}\right)=K\left(u_{1}, \cdots, u_{m}, u_{1}^{\prime}, \cdots, u_{m}^{\prime}, x_{1}, \cdots, x_{m}\right)$ and hence also the relation $K\left(u_{1}, \cdots, u_{m}, u_{1}^{\prime}, \cdots, u_{m}^{\prime}, x_{1}, \cdots, x_{m}\right)=K\left(u_{1}, \cdots, u_{m}\right.$, $\left.u_{1}^{\prime}, \cdots, u_{m}^{\prime}, x_{1}^{\prime}, \cdots, x_{m}^{\prime}\right)$. This shows that the Abelian variety $T_{m}(A)$ satisfies the hypothesis of Theorem 3, Corollary $1^{\prime}$, and hence there exists an Abelian variety $A^{\prime}$ over $K$, uniquely determined up to a regular isomorphism over $K$, such that for every sufficiently large in teger $m$, there is a regular isomorphism $R_{m}$ of $A^{\prime}$ onto $T_{m}(A)$. We set $F=p r_{1} R_{m}$.

Consider now the rational homomorphism $H$ of $B$ into $A^{*}$, and let $w$ be a generic point of $B$ over $K_{1}\left(u_{1}, \cdots, u_{m}\right)$, where we now assume that $K\left(u_{1}, \cdots, u_{m}\right)$ is independent with respect to $K_{1}$ over $K$; according to Theorem 7 , the point $H(w)$ is contained in $A_{u}$, hence there exists a point $y_{0}$ in $A$ such that $T_{u}\left(y_{0}\right)=H(w)$. The correspondence $w \rightarrow S\left[T_{u}^{-1}(H(w))\right]-S\left[T_{u}^{-1}(0)\right]$ defines a rational homomorphism of $B$ into $A$ (where the symbol $S$ has the meaning given in Weil [6, pp. 28-29]), defined over $K_{1}(u)$; according to Theorem 2, Corollary 1, this homomorphism is also defined over $K_{1}$ and hence $S\left[T_{u}^{-1}(H(w))\right]-S\left[T_{u}^{-1}(0)\right]$ is a rational point over $K_{1}(w)$ in $A$. Furthermore, the points in $T_{u}^{-1}(0)$ constitute a finite subgroup in $A$, normally algebraic over $K(u)$; according to Theorem 1 , this subgroup is also algebraic over $K$. It follows then that the point $y_{1}=S\left[T_{u}^{-1}(H(w))\right]$ is algebraic over $K_{1}(w)$. If $d$ is the degree of $T_{u}$, then we have $y_{1}=S\left[T_{u}^{-1}(H(w))\right]=S\left[T_{u}^{-1}\left(T_{u}\left(y_{0}\right)\right)\right]=d y_{0}$; it follows then that the point $y_{0}$ is algebraic over $K_{1}\left(y_{1}\right)$ and hence also algebraic over $K_{1}(w)$.

Now, for each $i=1, \cdots, m$, let $H_{u_{i}}$ be the specialization of $H$ over the specialization $u \rightarrow u_{i}$ over $K_{1}$; then $H_{u_{i}}$ is a rational homomorphism of $B$ into $A_{u_{i}}$, and since $K_{1}\left(u_{i}\right)$ and $K_{1}\left(w, y_{0}\right)$ are linearly disjoint over $K_{1}$, we have the relation $H_{u_{i}}(w)=T_{u_{i}}\left(y_{0}\right)$. The correspondence $w \rightarrow\left(H_{u_{1}}(w), \cdots, H_{u_{m}}(w)\right)=$ $\left(T_{u_{1}}\left(y_{0}\right), \cdots, T_{u_{m}}\left(y_{0}\right)\right)$ then defines a rational homomorphism $H_{m}$ of $B$ into $T_{m}(A)$, defined over $K\left(u_{1}, \cdots, u_{m}\right)$; and for sufficiently large $m$, we have then $H(w)=p r_{1} H_{m}(w)=p r_{1} R_{m} R_{m}^{-1} H_{m}(w)=F R_{m}^{-1} H_{m}(w)$. Since $R_{m}^{-1} H_{m}$ is a rational homomorphism of $B$ into $A^{\prime}$, defined over $K_{1}\left(u_{1}, \cdots, u_{m}\right)$, it is also defined over $K_{1}$, according to Theorem 2, Corollary 1 . This concludes the proof of our theorem.

The Abelian variety $A^{\prime}$ in Theorem 8 is called the $K$-trace of $A^{*}$ over $K^{*}$, and the rational isomorphism $F$ is called the canonical isomorphism of $A^{\prime}$ into 
$A^{*}$. The $K$-trace $A^{\prime}$ of $A^{*}$ over $K^{*}$ is uniquely determined up to a regular isomorphism over $K$, and the canonical isomorphism $F$ of $A^{\prime}$ into $A^{*}$ is uniquely determined up to regular isomorphisms of $A^{\prime}$ and $A_{0}{ }^{*}$ over $K$ and $K^{*}$ respectively, where $A_{0}{ }^{*}$ is the $K$-maximal Abelian subvariety over $K^{*}$ in $A^{*}$. If $K_{1}$ is any extension of $K$, independent with respect to $K^{*}$ over $K$, then the $K$-trace $A^{\prime}$ of $A^{*}$ over $K^{*}$ is also the $K_{1}$-trace of $A^{*}$ over $K^{*} K_{1}$, with the same canonical isomorphism $F$; furthermore, it is easily seen from Theorem 2, Corollary 1 , and the proof of Theorem 8 that if $K_{1}^{*}$ is any regular extension of $K^{*}$, then the $K$-trace $A^{\prime}$ of $A^{*}$ over $K^{*}$ is also the $K$-trace of $A^{*}$ over $K_{1}^{*}$, with the same canonical isomorphism $F$. In fact, in order that the latter statement holds, it is sufficient to assume that $K_{1}^{*}$ is a primary extension of $K^{*}$ and is also a regular extension of $K$.

Corollary. Let $K^{*} K(u)$ be a regular extension of $K$, and let $A^{*}$ be an Abelian variety over $K^{*}$; let $A^{\prime}$ be the $K$-trace of $A^{*}$ over $K^{*}$, and let $F$ be the canonical isomorphism of $A^{\prime}$ into $A^{*}$. Let $u_{1}, \cdots, u_{m}$ be independent generic specializations of $u$ over $K$, and let $A_{1}{ }^{*}, \cdots, A_{m}^{*}$ and $F_{1}, \cdots, F_{m}$ be the corresponding specializations of $A^{*}$ and $F$ respectively; if $y$ is a generic point of $A^{\prime}$ over $K\left(u_{1}, \cdots, u_{m}\right)$, then the correspondence $y \rightarrow\left(F_{1}(y), \cdots, F_{m}(y)\right)$ defines $a$ regular isomorphism of $A^{\prime}$ into the product variety $\prod_{i=1}^{m} A_{i}{ }^{*}$, provided the integer $m$ is taken sufficiently large.

This follows almost immediately from the proof of Theorem 8 .

There arises naturally the question whether a result similar to this corollary also holds for the $K$-image $A$ of $A^{*}$ over $K^{*}$, that is, in the notation of Theorem 4, whether the correspondence $\left(x_{1}, \cdots, x_{m}\right) \rightarrow \sum_{i=1}^{m} F_{i}\left(x_{i}\right)$ defines a regular homomorphism of $\prod_{i=1}^{m} A_{i}^{*}$ onto $A$, if the integer $m$ is taken sufficiently large. If this is true, then it would follow that, in case $K^{*}$ is a regular extension of $K$, the $K$-image of $A^{*}$ over $K^{*}$ is also the $K_{1}$-image of $A^{*}$ over $K^{*} K_{1}$ for any extension $K_{1}$ of $K$ which is independent with respect to $K^{*}$ over $K$. That the answer to this question is in the affirmative will be proved in our forthcoming paper on Picard varieties, as an application of the principle of duality developed there; however, it would be interesting and desirable to have a direct proof of this fact by the methods used in this paper.

Notes. (Added in proof, January 8,1955 .) (1) Professor Weil has kindly communicated to us a new, simpler proof of our Theorem 1 in $\$ 1$, based on the Theorem 7 and Proposition 25 in his book [6]. Using the notations in the proof of Theorem 1 , as well as the simplifying assumptions introduced there, Weil's proof can be summarized as follows. Let $W$ be a subvariety of dimension $r-d$ ( $r$ and $d$ being the dimensions of $A$ and $X$ respectively) in $A$, defined over $K$, having at 0 a simple point and transversal to $X$ at 0 ; then, according to [6, Proposition 25], the correspondence $y \rightarrow S\left(X \cdot W_{y}\right)$ defines up to a translation a rational homomorphism $H$ of $A$ onto $X$, defined over $K(u)$. On the other hand, the correspondence $y \times u \rightarrow H(y)$ defines a rational trans- 
formation $F$ of $A \times U$ into $A$, defined over $K$; according to [6, Theorem 7], we have $F(y, u)=F_{0}(y)+F_{1}(u)$, where $F_{0}$ and $F_{1}$ are rational transformations of $A$ and $U$ respectively into $A$, both defined over $K$. It follows then that we must have $H(y)=F_{0}(y)-F_{0}(0)$, so that $H$ is also defined over $K$, and this shows that $X$ as the image variety of $H$ is defined over $K$.

(2) We should like to make a few remarks on the proof of Theorem 3 in $\S 3$; for the sake of simplicity, we shall limit ourselves to the case where $K(v)$ is a regular extension of $K$, i.e. the case dealt with in the Corollary 1 , so that the existence of $A^{*}$ is not needed. We recall that, in the proof of Theorem 3, the following construction is used to obtain an Abelian variety defined over $K$ which is regularly isomorphic over $K(v)$ to a given Abelian variety $A$ defined over $K(v)$, after we have shown that there exists an Abelian variety $A_{1}^{\prime}$ defined over an algebraic extension $K\left(v_{1}^{\prime}\right)$ of $K$ which is regularly isomorphic to $A$ over $K\left(v, v_{1}^{\prime}\right)$ : Let $v_{1}^{\prime}, \cdots, v_{d}^{\prime}$ be the complete set of conjugates of $v_{1}^{\prime}$ over $K$, and let $A_{1}^{\prime}, \cdots, A_{d}^{\prime}$ be the corresponding specializations of $A_{1}^{\prime}$ over $K$; let $y$ be a generic point of $A$ over $K(v)$, and let $y_{1}^{\prime}, \cdots, y_{d}^{\prime}$ be the corresponding generic points of $A_{1}^{\prime}, \cdots, A_{d}^{\prime}$ over $K\left(v, v_{1}^{\prime}\right), \cdots, K\left(v, v_{d}^{\prime}\right)$ respectively; finally, let $z$ be the associated point of the 0 -cycle $\sum_{i=1}^{d}\left(y_{i}^{\prime}\right)$ as a cycle in the ambient projective space. Since $z$ is rational over $K(v, y)$, it has a locus $V$ over $K(v)$; then, under the additional assumptions of Theorem 3, it is shown that $V$ is an Abelian variety defined over $K$ and is regularly isomorphic to $A$ over $K(v)$. Professor Weil has kindly called our attention to the fact that a similar construction has been used by $\mathrm{T}$. Matsusaka in his paper Some theorems on Abelian varieties (Natural Science Report, Ochanomizu University, vol. 4, 1953, pp. 22-35), in the proof of his main theorem (Theorem 3), for a similar purpose; in his case, the variety $A$ is already defined over $K$, but is assumed to have only a normal law of composition, and the object is to prove that it is birationally equivalent to an Abelian variety defined over $K$. This method of construction seems to be useful also for other problems where one wishes to narrow down the field of definition of a certain type of varieties; in fact, in a paper to be published soon in the American Journal of Mathematics, Weil has applied this method in his theory of "transformation spaces" and succeeds in generalizing to abstract varieties some of the results of Matsusaka and ourselves.

In this connection, we should like to add the following comments, which will throw some further lights on the relation between the main result of Matsusaka's paper and our Theorem 3. If we remove from both the hypothesis and the conclusion of Theorem 3, or rather Corollary 1 of Theorem 3, the condition that the function fields involved are Abelian function fields, then the theorem will still be true, for we need only to omit in the proof all references to group properties. In fact, in this case, a much simpler proof of the theorem can be given as follows, using the notations in the proof of Theorem 3, whereby we shall again restrict ourselves to the case of Corollary 
1 , for the sake of simplicity. Let $X$ and $W$ be the loci of $\left(y^{\prime}, v^{\prime}\right)$ and $v^{\prime}$ over $K$ respectively; the condition $K\left(v, v^{\prime}, y\right)=K\left(v, v^{\prime}, y^{\prime}\right)$ shows that there is a birational correspondence $T$ of the product variety $A \times W$ onto $X$, defined over $K(v)$. Since $y \times W$ is a subvariety in $A \times W$, defined over $K(v, y)$, and since it contains a point $\left(y, v^{\prime}\right)$ which is a generic point of $A \times W$ over $K(v)$, the image variety $T(y \times W)$ is defined and is a prime rational cycle over $K(v, y)$. If $z$ is the associated point of $T(y \times W)$, then the correspondence $y \rightarrow z$ defines a rational transformation $R$ of $A$, defined over $K(v)$, which can be easily seen to be birational. Since the generic point $v$ of $W$ can be replaced by any other generic point, and since $K(v)$ is assumed to be regular over $K$, it follows that the image variety $V=R(A)$ must be defined over $K$; furthermore, if we now add the assumption that $A$ is Abelian, then the group composition in $A$ induces on $V$ a normal law of composition, which can be easily seen to be also defined over $K$. Thus we have given a new proof of our Theorem 3, with however the weaker conclusion that the variety $V$ has a normal law of composition, instead of being an Abelian variety; this was, in fact, our first (incomplete) proof of this theorem, and it is precisely in order to obtain the stronger conclusion, that $V$ is an Abelian variety, that we introduced an algebraic specialization of $A^{\prime}$ and applied the method of construction outlined above. Now, the main theorem in Matsusaka's paper (which unfortunately was not known to us at the time when this paper was written) asserts that any variety with a normal law of composition defined over $K$ is birationally equivalent over $K$ to an Abelian variety defined over $K$, and this is exactly what we need in order to complete the above argument into a proof of our Theorem 3, at least in the case of Corollary 1. This new proof, though in reality longer and more complicated than the one given in the text, considering the length of Matsusaka's proof of his main theorem, has nevertheless the advantage of conceptual simplicity.

(3) As we have observed after Theorem 4, the $K$-image $A$ of $A^{*}$ over $K^{*}$ remains to be the $K$-image of $A^{*}$ over $K_{1}^{*}$ for any primary extension $K_{1}^{*}$ of $K^{*}$. Professor Matsusaka has kindly pointed out to us that this is in a sense the exact extent to which the $K$-image is independent of the choice of the reference field $K^{*}$, and that a counter example can be constructed where $K_{1}^{*}$ is even a regular extension of $K$, though of course no longer a primary extension of $K^{*}$. A simple special case of the example communicated by Matsusaka is the following. Let $K$ be an algebraically closed field of characteristic zero, let $u$ be a variable over $K$, and consider the elliptic curve $C(u)$ in the plane defined by the equation $y^{2}=u f(x)$ over the field $K(u)$, where $f(x)$ is a polynomial of degree 4 (or 3 ) with coefficients in $K$ and having no multiple roots; since $C(u)$ has evidently a rational point over $K(u)$, it is an Abelian variety over $K(u)$. If $u^{\prime}$ is another variable over $K$, independent with respect to $u$ over $K$, then the curve $C\left(u^{\prime}\right)$ cannot be birationally equivalent over $K\left(u, u^{\prime}\right)$ to the curve $C(u)$; for, if it were so, there would be a birational transformation 
of $C(u)$ onto $C\left(u^{\prime}\right)$, defined over $K\left(u, u^{\prime}\right)$, which leaves the variable $x$ invariant and hence must transform the variable $y$ by the correspondence $y \rightarrow\left(u^{\prime} / u\right)^{1 / 2} y$, which is in contradiction to the fact that birational transformation is defined over $K\left(u, u^{\prime}\right)$. This shows that the $K$-image of $C(u)$ over $K(u)$ is zero, i.e. reduces to a point, while on the other hand it is obvious that the $K$-image of $C(u)$ over $K\left(u^{1 / 2}\right)$ is the elliptic curve $C$ defined by the equation $y^{2}=f(x)$. This situation is further confirmed by the observation, based on some of the results in our forthcoming paper on Picard varieties, that the $K$-images of $C(u)$ over $K(u)$ and $K\left(u^{1 / 2}\right)$ are respectively the Albanese varieties of the surfaces in 3-space defined by the equations $y^{2}-z f(x)$ $=0$ and $y^{2}-z^{2} f(x)=0$ over $K$; the former is a rational surface and hence has a zero Albanese variety, while the latter is a ruled surface, birationally equivalent to the product of the curve $C$ with a projective line.

\section{REFERENCES}

1. W. L. Chow, On Picard varieties, Amer. J. Math. vol. 74 (1952) pp. 895-909.

2. - On the quotient variety of an Abelian variety, Proc. Nat. Acad. Sci. U.S.A. vol. 38 (1952) pp. 1039-1044.

3. W. L. Chow and B. L. van der Waerden, Zur algebraischen Geometrie. IX. Ueber zugeordnete Formen und algebraische Systeme von algebraischen Mannigfaltigkeiten, Math. Ann. vol. 113 (1937) pp. 692-704.

4. S. MacLane, Modular fields, I. Separating transcendental bases, Duke Math. J. vol. 5 (1939) pp. 372-393.

5. A. Weil, Foundations of algebraic geometry, New York, 1946.

6. — Varietés abeliennes et courbes algebriques, Paris, 1948.

ThE Johns Hopkins University, BALTIMORE, MD. 